\title{
Enfermedad periodontal en pacientes diabéticos tipo 2 con y sin obesidad en Lima, Perú
}

Periodontal disease in type 2 diabetic patients with and without obesity from Lima, Perú

José Carlos Quezada Carrera ${ }^{1 a}$, Sonia Sacsaquispe Contreras ${ }^{1 b}$, Fredy Gutiérrez Ventura ${ }^{1 c}$, César del Castillo López ${ }^{1 d}$, Martha López Pinedo ${ }^{1 e}$

\section{RESUMEN}

Objetivos: Determinar si la enfermedad periodontal afecta más a pacientes diabéticos tipo 2 con obesidad que a los pacientes sin obesidad. Material y métodos: La población estuvo conformado por 170 pacientes con diagnóstico de Diabetes Mellitus (DM) tipo 2; fueron divididos en dos grupos 85 diabéticos obesos y 85 diabéticos no obesos: el grupo obesos fue de 55 mujeres y 30 hombres mientras que el grupo sin obesidad fue de 37 mujeres y 48 hombres. Se utilizó el Î́ndice Periodontal Comunitario Modificado y la ficha de registro de datos aprobados por la OMS, la evaluación clínica se realizó tomando en cuenta, profundidad de las bolsas y la presencia de furca dental. Resultados: De los 85 pacientes con obesidad, el 83,53\% presentó bolsas periodontales de 4-5 mm y el $16,47 \%$ presentó bolsas $\geq 6 \mathrm{~mm}$, mientras que en el grupo sin obesidad el 90,59\% presentó bolsas de $4-5 \mathrm{~mm}$, el $7,06 \%$ presentó bolsas $\geq 6 \mathrm{~mm}$ y el 2,35\% no presentó bolsas. Conclusión: No hay diferencias en los signos de enfermedad periodontal en pacinetes con diabetes mellitus tipo 2 con obesidad y sin obesidad.

PALABRAS CLAVE: Diabetes mellitus, inflamación, obesidad, enfermedad periodontal.

\section{SUMMARY}

Objectives: To determine the presence and characteristics of periodontal disease in patients with type 2 diabetes mellitus with and without obesity. Material and Methods: Population consisted of patients diagnosed with Diabetes Mellitus type $2(\mathrm{DM}) ; 170$ patients were divided among 85 obese diabetic patients and 85 diabetic patients not obese, obese patients 55 were women and 30 were men, in the non-obese group 37 were female and 48 male

Facultad de Estomatología Roberto Beltrán, Universidad Peruana Cayetano Heredia. Lima, Perú.

Diplomado en Medicina Oral y Cirugía Oral

Especialista en Medicina Oral y Patología Oral y Maxilofacial

Especialista en Cirugía Bucal y Maxilofacial.

Especialista en Salud Pública

Especialista en Periodoncia 
cases. Community Periodontal Index Modified and data recording sheet approved by WHO were used, clinical evaluation was performed taking into account, pocket depth and the presence of dental bifurcation. Results: Periodontal pockets of $4-5 \mathrm{~mm}$ in obese patients were $83.53 \%$ and $16.47 \%$ presented pockets $\geq 6 \mathrm{~mm}$, while in the group without obesity $90.59 \%$ showed pockets of $4-5 \mathrm{~mm}$ and $7.06 \%$ presented pockets $\geq 6 \mathrm{~mm}$ while $2.35 \%$ ( 2 cases) had no pockets. Conclusion: No differences in the signs of periodontal disease pacinetes with type 2 diabetes mellitus with obesity and non-obese.

\section{KEYWORDS: Diabetes mellitus, inflammation, obesity, periodontal disease.}

\section{INTRODUCCIÓN}

La diabetes mellitus (DM) es un problema sanitario y económico creciente en todo el mundo. En 2013, el número de persona con DM se estimó en 382 millones, en 2032 este número se elevará a 592 millones (1).

La DM es un grupo de enfermedades metabólicas caracterizadas por la hiperglucemia como resultado de defectos en la secreción de insulina, la acción de la insulina o ambos. Los pacientes con DM tienen una incidencia incrementada de arterioesclerosis vascular y enfermedades cerebrovasculares. La hipertensión también es un factor de riesgo en las personas con este desorden (2).

La periodontitis es una enfermedad crónica de la cavidad oral cuyos signos clínicos incluyen pérdida de inserción, pérdida de hueso alveolar, bolsas periodontales e inflamación gingival. Además también produce recesión gingival, sangrado, movilidad dentaria que puede llevar a la exfoliación dental (3).

Las características histopatológicas de la periodontitis incluyen la alteración del epitelio de unión hacia apical del límite amelocementario, pérdida de fibras colágenas, reabsorción ósea, infiltrado celular de células inflamatorias, células plasmáticas, linfocitos y macrófagos (3).

La proteína $\mathrm{C}$ reactiva (PCR) es una proteína plasmática implicada en la respuesta inflamatoria y se ha asociado con pérdida de hueso alveolar. La IL-1 también juega un papel fundamental en la inflamación crónica y elevados niveles de esta molécula han sido detectados en el fluido crevicular gingival de zonas con reciente pérdida ósea y de inserción en pacientes con periodontitis. La IL-6 sinergia con IL$1 \mathrm{~b}$ para mejorar el reclutamiento y la formación de osteoclastos, que inducen la resorción ósea. El TNF- $\alpha$ está involucrado en la regulación de la producción de colagenasa PGE2, quimiocinas, citosinas y la adhesión celular molecular, así como en la estimulación de la resorción ósea relacionada con estos factores (4).

Rojas realizó un estudio de indicadores de riesgo de salud oral en La Libertad - Perú, utilizando el índice periodontal comunitario de necesidad de tratamiento concluyó que los adolescentes presentaron una condición periodontal saludable estable fluctuando entre $6,3 \%$ y $10,8 \%$. Además la tendencia en la condición de sangrado es decreciente respecto a la edad, siendo a los 10 años con $69,2 \%$ y a los 15 años con $21 \%$, mientras que con respecto al cálculo dental la tendencia fue ascendente con $21,5 \%$ a los 10 años y $72,3 \%$ a los 15 años; en relación al sexo no se mostró diferencias significativas en la salud periodontal (5).

La hiperglicemia crónica de la diabetes está asociada con daño a largo plazo, disfunción, y falla de varios órganos, especialmente los ojos, riñones, nervios, corazón y venas (2).

La hemoglobina glicosilada A1c (HbAlc) se sintetiza en forma continua en los eritrocitos como producto de una reacción entre la proteína hemoglobina y la glucosa. El valor de la concentración de $\mathrm{HbAlc}$ tiene un valor clínico muy destacado que refleja con exactitud la glucemia media durante los últimos 1-3 meses (6).

El estudio epidemiológico realizado por Ramos y col en 18 hospitales peruanos, de los 2959 pacientes diagnosticados con DM tipo 1, el 29,8\% presentaba alguna complicación secundaria, las más frecuentes fueron la neuropatía $(21,4 \%)$, pie diabético $(5,9 \%)$ y nefropatía $(3,9 \%)(7)$.

La DM tipo 2 es un grupo de enfermedades metabólicas, es la forma más común, ocurre principalmente en adultos; aunque la prevalencia en gente joven está 
incrementándose en conjunción con la obesidad (8).

La mayoría de los pacientes que manifiestan este tipo de diabetes es obesa o tiene un aumento del porcentaje de grasa corporal distribuida principalmente en la región abdominal. El tejido adiposo tiene una importancia notable en la aparición de la resistencia a la insulina. (9).

Yang y col., examinaron 46239 adultos, en las zonas urbanas la prevalencia de DM en el sexo masculino fue de $12,8 \%$ y en el femenino fue de $10,1 \%$, y en las zonas rurales, la prevalencia fue de $8,9 \%$ y $7,7 \%$ respectivamente $(10)$.

López y col,. mediante una encuesta nacional en Colombia demostraron que la prevalencia de DM tipo 2 en la población entre los 18 y 69 años fue solo de $2,6 \%$ siendo mayor en hombres; asimismo comparó con el resultados de países latinoamericanos donde hay un rango de 5-12\% (11).

En el Perú al año 2011 la cantidad de pacientes era de 942 200, un 5,3\%, al año 2013 fue de 5,5\% y para el año 2030 será de 6,4\%. (12).

La obesidad es el trastorno metabólico más frecuente secundario al desequilibrio de energía, en el cual la ingesta supera al gasto de la misma, durante un periodo prolongado con consecuencias sociales, biológicas y mentales. A la epidemia mundial del sobrepeso y la obesidad, se le ha denominado "globesidad", coexistiendo paradójicamente con la desnutrición en los países en desarrollo (13).

Chescheir cita que la Organizaciòn Mundial de la Salud considera a la obesidad una epidemia crónica no transmisible que inicia a edades tempranas con un origen multicausal, y estima que en el 2015 se incrementará a 2,3 mil millones de personas con sobrepeso y 700 millones de personas obesas (14).

Álvarez y col., encontraron que el sobrepeso fue ligeramente mayor en hombres y la obesidad fue mayor en las mujeres; mayores en el área urbana y aumenta conforme disminuye el nivel de pobreza. Asimismo, se observó que fue más prevalente en Lima Metropolitana y la costa. A nivel regional, la prevalencia de la obesidad fue mayor en Madre de Dios (77,4\%); Tacna (75,0\%); Ica (73,1\%); Lima (70,4\%), y Tum- bes $(70,0 \%)$. Fue menos prevalente en Huancavelica $(36,0 \%)$, Apurímac (41,1\%), Huánuco (42,0\%), Amazonas (46,2\%) y Pasco (46,4\%) (15).

Colosia y col., evaluaron 2688 pacientes obesos de diferentes continentes, encontraron en Europa 50.9\% de DM tipo 2, en Asia fue de 56.1\% y en Estados Unidos fue de 58\% (16).

No hay muchos estudios que relacionan la asociación de obesidad y EP; sin embargo el tejido adiposo derivado de las citoquinas y hormonas, colectivamente llamadas adipoquinas o adipocitoquinas, podrían modular la EP. El sistema de activación plasminógeno tiene un rol importante en la inflamación gingival (17).

Sayed evaluó 380 pacientes, el 34\% fueron pacientes obesos y presentaban una pérdida de inserción de 2,1 $\mathrm{mm}$ concluyendo que estos pacientes tiene un alto riesgo de aseverar el grado de periodontitis (18).

Ariza concluye que la diabetes tipo 2 no controlada está asociada a periodontitis crónica lo cual indica que los pacientes con diabetes tipo 2 no controlada tienen 1,98 veces más probabilidad de presentar periodontitis que los pacientes diabéticos tipo 2 controlados (19).

Eldarrat afirma que la mayoría de participantes con DM tipo 2 presentan manifestaciones orales como EP (78\%), caries dental (54\%) y xerostomía (31\%), esto se debe principalmente por la falta de higiene ya que solo el $17 \%$ se cepilla los dientes 2 veces al día (20).

Ha sido propuesto que en la DM tipo 2 se da una respuesta inflamatoria del huésped por una continua fase aguda inducida por las citoquinas que están estrechamente relacionadas en la patogénesis de la enfermedad y sus complicaciones asociadas (21).

La DM trae consigo hiperglucemia la cual afecta la síntesis de fibroblastos, resultando en la pérdida de fibras periodontales y pérdida ósea (22).

\section{MATERIAL Y MÉTODOS}

El diseño del estudio fue transversal, cuantitativo y observacional.

La población estuvo constituido por pacientes con diagnóstico de DM tipo 2 atendidos en el Consultorio 
Externo en el Servicio de Endocrinología del Hospital Nacional Cayetano Heredia (HNCH) en el año 2014.

Se utilizó una muestra no aleatoria de 170 pacientes, obtenido del estudio realizado por Basukala (37), que ingresaron al Servicio de Endocrinología del $\mathrm{HNCH}$ en el año 2014 fueron divididos entre 85 pacientes diabéticos obesos y 85 pacientes diabéticos no obesos, al término de cada cita, se les abordó explicando los beneficios del estudios, luego fueron citados en los días posteriores a la Clínica Dental Docente Cayetano Heredia.

Fueron incluidospacientes con diagnóstico de DM tipo 2, con pesos normales y obesos que voluntariamente aceptaron las condiciones del estudio, fueron atendidos en el Consultorio Externo del Servicio de Endocrinología del HNCH en el año 2014. Se excluyeron mujeres gestantes, con alguna enfermedad sistémica $\mathrm{u}$ otros pacientes que fallaron en los criterios de exclusión

Fueron distribuidos de acuerdo a su IMC determinando así su condición, fueron examinados en los ambientes del servicio de odontología del $\mathrm{HNCH}$ y en los módulos dentales de la Clínica Dental Docente Cayetano Heredia, con el paciente sentado en posición de 90 grados, bajo luz de lámpara. Se utilizó, bajalenguas de madera desechables, espejos bucales y sondas periodontales.

Se utilizó el Índice Periodontal Comunitario Modificado y la ficha de registro de datos aprobados por la OMS para cada paciente donde se anotó el código de acuerdo al resultado obtenido. La decisión de elegir el IPC fue que, a diferencia del periodontograma general u otros índices, el IPC sólo evalúa profundidad de bolsas periodontales por pieza, más no por superficies o caras, por consiguiente la ejecución del protocolo fue más rápida ya que la presencia de bolsas justifica la presencia de enfermedad periodontal, si se requiriera comparaciones entre piezas dentarias de pacientes obesos y no obesos, la decisión correcta hubiera sido el periodontograma. Los pasos clínicos de evaluación se realizaron tomando en cuenta los atributos de la encía y de las piezas dentarias: profundidad de las bolsas y la presencia de furca dental.

Con la finalidad de uniformizar criterios en el diagnóstico clínico, se realizó un entrenamiento a través de la práctica clínica intensiva en el dominio de los índices periodontales a cargo de un docente del área de periodoncia asignado por la Facultad de Estomatología Roberto Beltrán de la Universidad Peruana Cayetano Heredia.

Plan de análisis: Se presentaron los datos mediante tablas de distribución de frecuencias y gráficos de barras. El análisis bivariado se realizó mediante la prueba Chi-Cuadrado para evaluar la asociación entre enfermedad periodontal y las personas con obesidad y sin ella.

Consideraciones éticas: La investigación se desarrolló después de la aprobación del Comité Institucional de Ética (CIE) de la Universidad Peruana Cayetano Heredia.

\section{RESULTADOS}

Se evaluó durante seis meses de abril - setiembre del año 2014, pacientes del servicio de endocrinología que cumplían los criterios de inclusión, 170 pacientes fueron evaluados los cuales fueron divididos entre 85 pacientes diabéticos obesos y 85 pacientes diabéticos no obesos.

De acuerdo al sexo, en el grupo de pacientes con obesidad el $64,71 \%$ (55 casos) son mujeres y el $35,29 \%$ (30 casos) son hombres mientras que en el grupo sin obesidad hay $43,53 \%$ ( 37 casos) del sexo femenino y $56,47 \%$ (48 casos) del sexo masculino

De acuerdo a la edad en el grupo de pacientes con obesidad el $50 \%$ presentaba rangos de edad entre 40 y 54 años de edad y el otro $50 \%$ consta de pacientes $\geq$ 60 años de edad; así también en el grupo sin obesidad el $50 \%$ presentaba un rango de edades entre 42 y 54 años de edad y el otro $50 \%$ presentan $\geq 60$ años de edad por lo que existe una relación estrecha entre la obesidad y los pacientes mayores de 60 años.

De acuerdo al IMC, el grupo con obesidad presentaba un IMC alrededor de $31,8 \pm 1,8 \mathrm{Kg} / \mathrm{m}^{2}$ de los cuales el 95,2\% (81 casos) presentaba obesidad tipo I, el $3,63 \%$ ( 3 casos) con obesidad tipo II y el 1,17\% (1 casos) con obesidad tipo III; mientras que en el grupo sin obesidad presentaban un IMC alrededor de 22,4 \pm $1,5 \mathrm{Kg} / \mathrm{m}^{2}$ (Tabla 1 y Gráfico 1 ). 
De acuerdo a la frecuencia de bolsas periodontales, los pacientes con obesidad el 83,53\% (71 casos) presentó bolsas de 4-5 $\mathrm{mm}$ y el 16,47\% (14 casos) presentó bolsas $\geq 6 \mathrm{~mm}$, mientras que en el grupo sin obesidad el 90,59\% (77 casos) presentó bolsas de 4-5 $\mathrm{mm}$, el 7,06\% (6 casos) presentó bolsas $\geq 6 \mathrm{~mm}$ y el $2,35 \%$ ( 2 casos) no presentó bolsas.

Con respecto al sangrado gingival, el grupo de pacientes con obesidad el 2,35\% (2 casos) presento ausencia de sangrado y el $97,65 \%$ (83 casos) con presencia de sangrado, mientras que en el grupo sin obesidad el 30,58\% (36 casos) presentó ausencia de sangrado gingival y el 69,42\% (49 casos) con presencia de sangrado gingival (Tabla 2 y gráfico 2 ).

La frecuencia de EP en ambos grupos, se observó la presencia de EP en el 100\% del grupo de pacientes con obesidad; mientras que en el grupo si obesidad, sólo es del 97,65\% (Tabla 3 y gráfico 3 ).

La severidad de las bolsas periodontales de los pacientes con obesidad es mayor que en los pacientes con peso normal; ambos grupos presentan ligeramente la misma cantidad de pacientes con bolsas periodontales de 4-5 mm, el grupo con obesidad presenta mayor cantidad de bolsas periodontales $\geq 6 \mathrm{~mm}$ y ningún paciente presentó ausencia de EP; se consideró clínicamente significativa, pero al aplicar la prueba de Fisher, se demuestra que no hay una diferencia estadísticamente significativa con respecto a la enfermedad periodontal en ambos grupos $(\mathrm{p}>0,05)$.

El sangrado gingival estuvo presente en ambos grupos superando en un $50 \%$, el grupo de pacientes con obe- sidad presentó un porcentaje aproximado al 100\% a diferencia del grupo con peso normal $(69,42 \%)$; la ausencia de sangrado gingival es muy baja en los pacientes con obesidad (2,35\%), hay una diferencia clínica significativa, aplicando la prueba de Fisher, se observa una diferencia estadísticamente significativa $(p<0,05)$.

En cuanto a las piezas dentarias afectadas en el grupo sin obesidad se observó en el 15,20\% de pacientes, quienes presentaron un promedio de 0 a 5 piezas afectadas con bolsas periodontales, el $22,35 \%$ presentó un promedio de 6 a 10 piezas, el $25.88 \%$ con 11 a 15 piezas y el $36,47 \%$ con un promedio de 16 a 18 piezas dentarias; en el grupo con obesidad se observó que la cantidad de pacientes con un promedio de piezas afectadas de 16 a 18 es de $40 \%$; el número de piezas afectadas es ligeramente igual en ambos grupos pero clínicamente las piezas dentarias de los pacientes con obesidad posee bolsas $\geq 6 \mathrm{~mm}$.

En cuanto a la severidad de EP, sólo el 15,29\% de pacientes del grupo normal presenta ausencia de 7 a 10 piezas dentarias; mientras que en el grupo obesidad sólo el 35,29\% presenta ausencia de 7 a 10 piezas dentarias; mientras que los pacientes del grupo normal presentan menor cantidad de piezas ausentes que los pacientes con obesidad. Con respecto a la arcada más prevalente, el sector inferior fue el más prevalente en contener mayor cantidad de piezas afectadas con bolsas periodontales en ambos grupos; esto se debe a la facilidad de las piezas dentarias inferiores en acumular placa dental a diferencia de las piezas dentarias superiores.

Tabla 1. Número de pacientes sin obesidad con obesidad y porcentaje

(\%) divididos de acuerdo al sexo, edad e IMC.

\begin{tabular}{lll}
\hline & $\begin{array}{c}\text { Sin Obesidad } \\
(\mathrm{n}=85)\end{array}$ & $\begin{array}{c}\text { Obesidad } \\
(\mathrm{n}=85)\end{array}$ \\
\hline Sexo & & $55(64,71)$ \\
Femenino & $37(43,53)$ & $30(35,29)$ \\
Masculino & $48(56,47)$ & $47 \pm 7,5$ \\
Edad* & $48 \pm 6,5$ & $31,8 \pm 1,8$ \\
IMC & $22,4 \pm 1,5$ &
\end{tabular}


Tabla 2. Número de pacientes sin obesidad con obesidad y porcentaje (\%) según el grado de bolsas periodontales y sangrado gingival de acuerdo al índice periodontal comunitario (OMS).

\begin{tabular}{llll}
\hline & $\begin{array}{l}\text { Sin obesidad } \\
(\mathrm{n}=85)\end{array}$ & $\begin{array}{l}\text { Obesidad } \\
(\mathrm{n}=85)\end{array}$ & valor de $\mathrm{p}^{*}$ \\
\hline $\begin{array}{l}\text { Bolsas Periodontales } \\
\text { Ausencia }\end{array}$ & $2(2,35)$ & $0(0,0)$ & \\
$\begin{array}{l}\text { Presencia } \\
4-5 \mathrm{~mm}\end{array}$ & $77(90,59)$ & $71(83,53)$ & \\
$\geq 6 \mathrm{~mm}$ & $6(7,06)$ & $14(16,47)$ & 0,04 \\
& & & \\
\multicolumn{1}{c}{ Sangrado Gingival } & & & \\
Ausencia & $26(30,58)$ & $2(2,35)$ & \\
Presencia & $59(69,42)$ & $83(97,65)$ & 0,066 \\
\hline
\end{tabular}

*Prueba exacta de Fisher

Tabla 3. Número de pacientes sin obesidad con obesidad y porcentaje (\%) según la presencia de EP de acuerdo al índice periodontal comunitario (OMS).

\begin{tabular}{llcl}
\hline & $\begin{array}{c}\text { Sin obesidad } \\
(\mathrm{n}=85)\end{array}$ & $\begin{array}{c}\text { Obesidad } \\
(\mathrm{n}=85)\end{array}$ & valor de $\mathrm{p}^{*}$ \\
\hline EP & $2(2,35)$ & $0(0)$ & \\
Ausencia & $83(97,65)$ & $85(100)$ & 0,03 \\
Presencia & & \\
\hline
\end{tabular}

*Prueba exacta de Fisher 


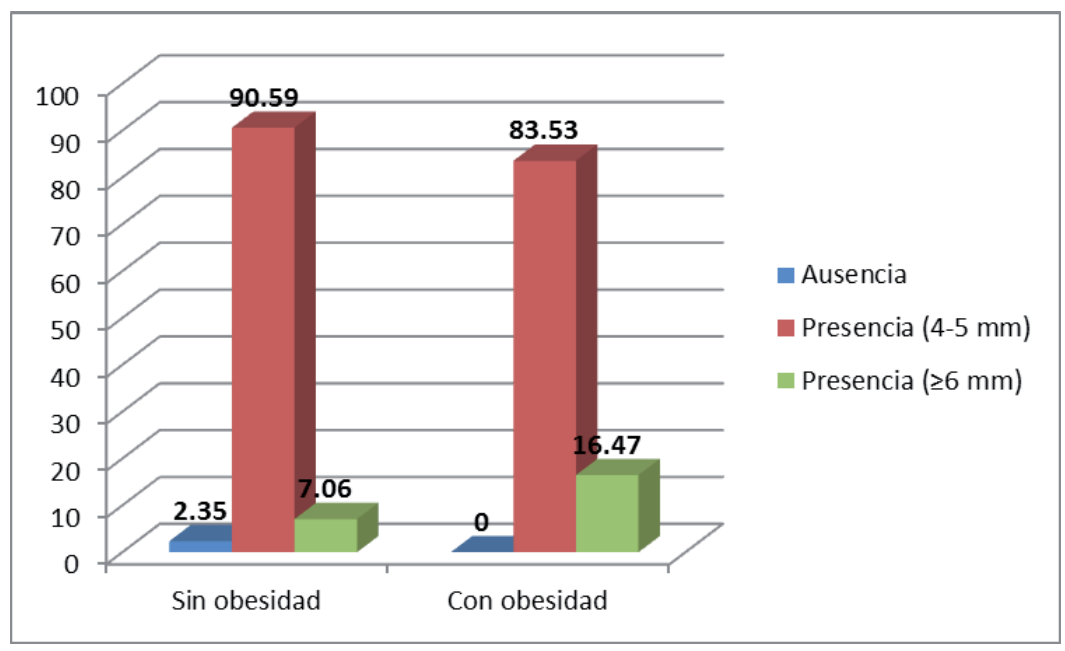

Gráfico 1. Presencia de bolsas periodontales en pacientes diabéticos tipo 2

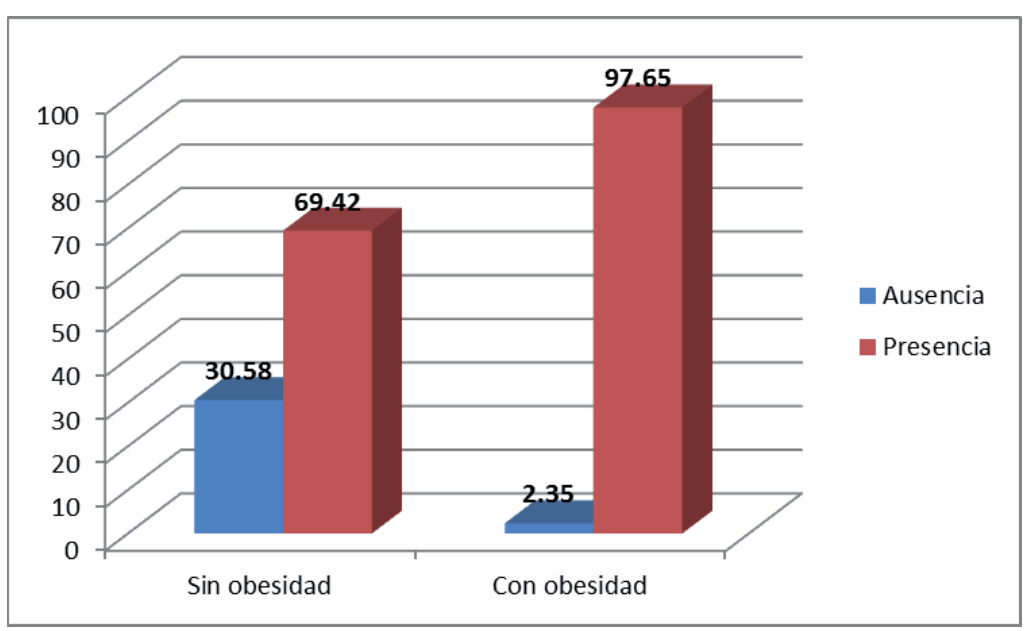

Gráfico 2. Presencia de Sangrado gingival en pacientes diabéticos tipo 2

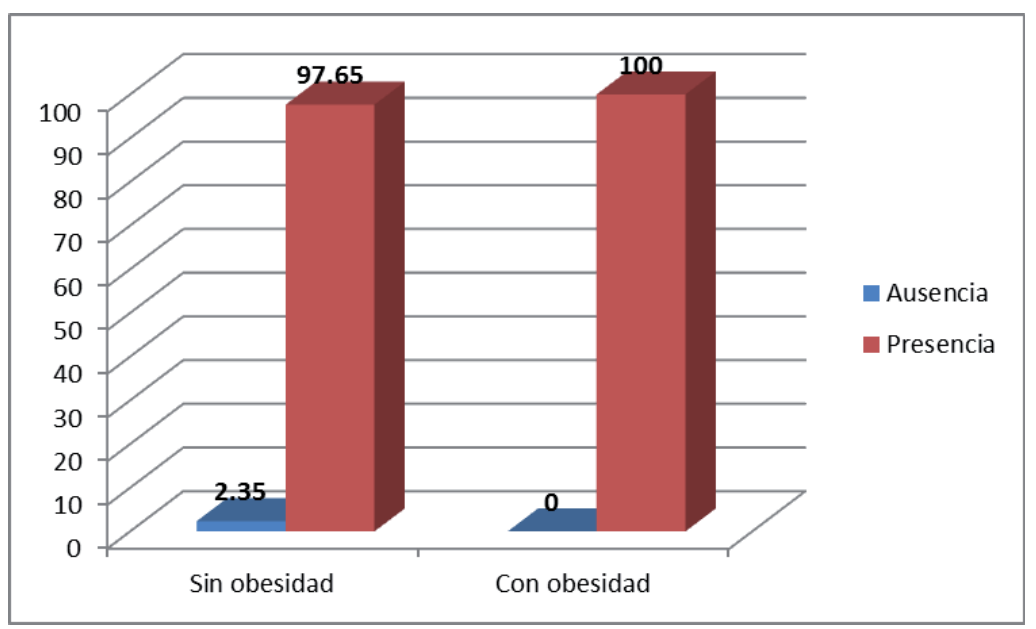

Gráfico 3. Presencia de EP en pacientes diabéticos tipo 2 


\section{DISCUSIÓN}

Novak M y col. evaluaron una población de estadounidenses de origen hispano con DM tipo 2 con obesidad en un rango de edades de 35 a 72 años, los cuales presentaban mayor cantidad de placa supra y subgingival, mayor sangrado gingival $(77,4 \%)$ así como un aumento en la extensión y severidad de la destrucción periodontal, así como el aumento de la frecuencia del número de dientes perdidos debido a la periodontitis confirmando que la EP está presente en un $57,5 \%$ y que la obesidad es un factor de riesgo para la periodontitis. Este resultado respalda parte de la investigación ya que en el rango de edades entre 30-65 años de edad, la prevalencia de sangrado gingival fue de $97.65 \%$ debido al incremento de células inflamatorias por la DM tipo 2 volviendo más susceptible al pacientes a distintos grados de inflamación pero difieren con nuestro resultado en EP de 90,59\% debido al rango de edades utilizados hasta 72 años ya que estos pacientes presentan mayor ausencia de piezas dentarias disminuyendo así el número de piezas evaluadas (23).

Morita y col. estudiaron pacientes con DM tipo 2 en el año 2011 en el cual obtuvieron para los varones con $\mathrm{IMC} \geq 30$ que no hay diferencia significativa $(\mathrm{p}>0,05)$ para desarrollar la enfermedad periodontal aunque la presencia de periodontitis fue mayor en mujeres que en hombres. Los resultados concuerdan con nuestro estudio ya que de la variable EP tampoco obtuvo resultados con diferencias significativas (24).

Morita y col. estudiaron una población japonesa de 2478 pacientes para evaluar la presencia de periodontitis y el índice de masa corporal en pacientes con DM tipo 2, este estudio determinó que si existe asociación entre ambas situaciones. Estos resultados difieren totalmente del nuestro ya que no se encontró diferencias significativas, esto es debido a la diferencia abrumadora entre ambas muestras, otro factor que podría causar esta diferencia es la comparación entre la raza hispana y asiática (25).

Resultado distinto al de Chaffe y col., quienes realizaron un meta análisis en pacientes con DM tipo 2 con obesidad y EP en el cual concluyeron que a pesar de los pocos estudios de calidad se obtuvo una diferencia significativa $(p<0,005)$ en la asociación entre
EP y obesidad dentro de los 142 artículos encontrados en bases de datos académicas pero se evidencia una incapacidad para distinguir entre el orden temporal de los acontecimientos, lo que limita la evidencia de que la obesidad es un factor de riesgo para la enfermedad periodontal o que la periodontitis puede aumentar el riesgo de aumento de peso (26). En el presente estudio los pacientes no presentaron una diferencia significativa comparada a los resultados obtenidos por Chaffe.

La prevalencia de EP en pacientes obesos diabéticos tipo 2 según Awuti y col., fue de 47,1\% (453 de 962 individuos); bolsas de $4 \mathrm{~mm}(28,9 \%)$, de $5-6 \mathrm{~mm}$ $(10,2 \%)$ y $>6 \mathrm{~mm}(8,0 \%)$ respectivamente, los resultados son diferentes a nuestra investigación ya que se obtuvo a la EP distribuida en pacientes con bolsas de $4-5 \mathrm{~mm}(83,53 \%)$ y $16,47 \%$ con bolsas $\geq 6 \mathrm{~mm}$ debido a la diferencia entre ambas muestras (27).

Dumitrescu y col., realizaron un estudio similar la presente investigación en una muestra entre 79 pacientes obesos y 79 pacientes con peso normal, ambos grupos con DM tipo 2, la EP con bolsas de $4-5$ $\mathrm{mm}$ se presentaba en un $65 \%$ en los pacientes con IMC mayor a 30 (obesidad); el estudio resulta ser semejante al presente trabajo, un $83,53 \%$ obtenido en los pacientes con obesidad con bolsas de $4-5 \mathrm{~mm}$, esto es debido a la similitud casi exacta entre ambas muestras (28).

Otro estudio hecho por Kongstad y col., evaluó a 878 pacientes mujeres con un promedio de edad entre 20 y 75 años divididos entre obesas y no obesas donde les realizaron exámenes completos de la cavidad bucal concluyeron que el $60 \%$ de personas obesas tienen un grado de EP de grado moderada (bolsas periodontales $\geq 4 \mathrm{~mm}$ ), nuestro estudio difiere totalmente de la presente investigación ya que en el total de las 92 pacientes mujeres con promedio de edad entre 40 y 70 años divididos entre obesas y no obesas se obtuvo que el $92,52 \%$ (51 casos) presentaba bolsas de 4-5 mm y el $7,48 \%$ (4 casos) presentaba bolsas $\geq 6 \mathrm{~mm}$, por lo que el rango de edades, la muestra y el resultado final difieren totalmente a nuestros resultados (29).

Wolff y col., demostraron que el predominio de EP es mayor en el sexo masculino con un IMC superior a 30 y DM tipo $2(68 \%)$ con un promedio de 50,9 años de edad mientras que en el sexo femenino, la princi- 
pal manifestación fue el sangrado gingival (32\%); en la presente investigación la EP fue mayor (bolsas de $4-5 \mathrm{~mm} 83,53 \%$ ) y el sangrado gingival $(97,65 \%)$ con una edad promedio de 48 años en pacientes con obesidad y DM tipo 2; ambos estudios difieren ya que la muestra utilizada por Wolff sólo fue de 59 pacientes en total (30).

El estudio realizado por Martínez y col., en 66 pacientes diabéticos tipo 2 del servicio de endocrinología de un hospital nacional con obesidad concluyó que la EP está presente en el $51 \%$ de pacientes femeninos con bolsas $\geq 6 \mathrm{~mm}$ y el $58 \%$ de pacientes masculinos con bolsas $\geq 6 \mathrm{~mm}$ en el rango de edades entre 41 y 70 años de edad; estos resultados difieren al $16,47 \%$ de pacientes con bolsas periodontales $\geq 6$ $\mathrm{mm}$ de la presente investigación, esto es debido a la muestra utilizada por Martínez (31).

Otro estudio realizado en una clínica especialidad en diabetes por Prior D. en pacientes con DM tipo 2 y obesidad en una muestra de 103 pacientes con un promedio de edad de 55 años, la presencia de bolsas periodontales de $4-5 \mathrm{~mm}$ fue de $79.6 \%$; este resultado es similar al $83.53 \%$ que se obtuvo en la presente investigación; esto es debido a que en el artículo, los pacientes no habían recibido ningún tratamiento dental desde hace 1 año hasta la fecha a pesar de la diferencia entre ambas muestras (32).

\section{CONCLUSIONES}

No hay diferencias en los signos de enfermedad periodontal en pacinetes con diabetes mellitus tipo 2 con obesidad y sin obesidad.

\section{RECOMENDACIONES}

Realizar profilaxis dental en los pacientes que lo requieran ya que la placa dental puede impedir una adecuada recolección de datos y aumentar el tiempo de trabajo.

Aumentar la muestra brindará mejores resultados en futuros trabajos de investigación similares al presente estudio.

Evaluar el tiempo que el paciente presenta la DM tipo 2 o si ésta se encuentra controlada son factores que deben ser evaluados previamente.

Trabajar en conjunto con los asistentes de ambos servicios facilitará el trabajo y el resultado final del trabajo.

\section{Correspondencia:}

José Carlos Quezada Carrera

Correo electrónico: jose.quezada@upch.pe

\section{REFERENCIAS BIBLIOGRÁFICAS}

1. Han N, Whiting D, Guariguara L, Aschner P, Forouhi N. Atlas de la diabetes de la FD. 6ta edición. Barcelona: FID; 2013. p. 145

2. American Diabetes Association. Diagnosis and classification of diabetes mellitus. Diabetes Care. 2013;36(1):67-74.

3. Flemming T. Periodontitis. Ann Periodontol. 1999;4(1):32-34.

4. Aspirello S, Zizzi A, Tirabassi G, Buldreghini E, Biscotti T, Faloia E. Diabetes mellitus - associated periodontitis: differences between type 1 and type 2 diabetes mellitus. J Periodontol Res. 2011;46(1):164-69.

5. Rojas A, Pachas F. Perfil epidemiológico de salud oral e indicadores de riesgo en escolares adolescents de la localidad de Cartavio (La Libertad, Perú). Rev Estomatol Herediana. 2010; 20(3):127-36.

6. Mealey B, Ocampo G. Diabetes mellitus and periodontal disease. Periodontology. 2009;44(1):127-53.

7. Ramos W, López T, Revilla L, More L, Huamaní M, Pozo M. Resultados de la vigilancia epidemiológica de diabetes mellitus en hospitales notificantes del Perú, 2012. Rev Peru Med Exp Salud Publica. 2014; 31(1):9-15.

8. Hernández C. Interrelación entre diabetes, obesidad y enfermedad periodontal. Rev Mex Periodontol. 2011;2(1):7-11.

9. American Diabetes Association. Standars of medical care in diabetes. Diabetes Care. 2014;37(1):11-61.

10. Yang W, Lu J, Weng J, et al. . Prevalence of diabetes among men and women in China. N Engl J Med. 
2010;362(12):1090-101.

11. López P, Rey J, Rodríguez Y, López J. Epidemia de diabetes mellitus tipo 2 en latinoamerica. Medicina. 2010; 4(91):308-21.

12. Revilla L. Situación de la vigilancia de diabetes en el Perú al primer semestre del 2013. Bol. Epidemiol. 2013; 22(39):825-28.

13. Ortiz R, Nava G, Muñoz S, Veras M. Epidemiología de la obesidad. En: Ortiz R, Nava G, Muñoz S, Veras M. besidad un enfoque multidisciplinario. 1ra edición. Hidalgo: Ciencia al día; 2010 .p. 75-99.

14. Chescheir N. Obesidad en el mundo y su efecto en la salud de la mujer. Obstet Gynecol. 2011;117(1):121322.

15. Álvarez D, Sánchez J, Gómez G, Tarqui C. Sobrepeso y obesidad: prevalencia y determinantes sociales del exceso de peso en la población peruana (2009-2010). Rev Perú Med Exp Salud Pública. 2012;29(3):303-13.

16. Colosia A, Palencia R, Khan S. Prevalence of hypertension and Obesity in patients with type 2 diabetes mellitus in observational studies: a systematic literature review. Diabetes Metab Syndr Obes. 2013; 6(1): 327-38.

17. Navarrete C, Cartes R. Prevalencia de diabetes tipo 2 y obesidad en comunidades Pehuenches, Alto Biobio. Rev Chil Nutr. 2012;39(3):7-10.

18. Sayed H. Relationship between overall and abdominal obesity and periodontal disease among young adults. EMHJ. 2010;16(4):429-33.

19. Ariza T. Asociación entre periodontitis crónica y diabetes mellitus tipo 2 en pacientes controlados y no controlados atendidos en el consultorio externo del servicio de endocrinología del Hospital Nacional Cayetano Heredia del año 2011. Tesis de Magister. Lima, Perú: Universidad Peruana Cayetano Heredia; 2012. 46-55 pp.

20. Eldarrat A. Awareness and attitude of diabetic patients about their increased risk for oral diseases. Oral Health Prev Dent. 2011;9(3):235-41.

21. Santos R, Foss M, Rocha G. Impact of periodontitis on the diabetes related inflammatory status. J Can Dent Assoc. 2010;76(1):1-35.

22. Choi Y, McKeown R, Mayer E, Liese A, Song K, Merchant A. Association between periodontitis and impaired fasting glucose and diabetes. Diabetes Care. 2011;34(2): 381-86.

23. Novak J, Potter R, Blodgett J, Ebersonle J. Periodontal disease in Hispanic Americans with type 2 diabetes. J Periodontal. 2009;79(4):629-36.

24. Morita I. Five year incidence of periodontal disease in related to body mass index. J Dent Res. 2011;90(2):199-2.

25. Morita T. Association between periodontal disease and metabolic syndrome. J Public Health Dent. 2009;69(4):248-53.
26. Chaffe B, Weston S. Association between chronic periodontal disease and obesity: a systematic review and meta-analysis. J Periodontol. 2010;81(12):170824.

27. Awuti G, Younusi K, Linlin L, Upur H, Ren J. Epidemiological survey on the prevalence of periodontitis and diabetes mellitus in Uyghur adults from rural hotan area in Xinjiang. Exp Diabetes Res. 2012;10(12):1-7.

28. Dumitrescu A, Kawamura M. Involvement of psychosocial factors in the association of obesity with periodontitis. J Oral Sci. 2010;52(1):115-24.

29. Kongstad J, Hvidtfeldt U, Gronbaek M, Stoltze K, Holmstrup P. The relationship between body mass index and periodontitis in the Copenhagen City Heart study. J Periodontol. 2009;80(8):1246-53.

30. Wolff R, Wolff L, Michalowicz B. A pilot study of glycosylated hemoglobin levels in periodontitis cases and healthy controls. J Periodontol. 2009;80(7):1057-61.

31. Martínez A, González F, Nicolau O, Suárez B. Manifestaciones orales en portadores de diabetes mellitus tipo 2 de reciente diagnóstico. Arch Med Camaguey. 2010;14(1):1-14.

32. Prior P. Obesidad central, porcentaje de grasa corporal y enfermedad periodontal en pacientes con diabetes tipo 2 de la clínica de diabetes de Actopan, Hidalgo. Tesis Bachiller. Facultad de Nutrición, Universidad Autónoma del Estado de Hidalgo; 2008.37-40 pp.

33. World Health Organization. WHO Expert Consultation on Obesity: Preventing and Managing the Global Epidemic. 3raedición. Ginebra: WHO;2004. p. 94-110.

34. American Diabetes Association. Diagnosis and classification of diabetes mellitus. Diabetes Care. 2013;36(1):67-74.

Recibido : 05/05/2015 Aceptado: 15/07/2015 